\title{
Nocturnal Enuresis is an Under-recognised Side Effect of Clozapine: Results of a Systematic Review
}

\author{
${ }^{1}$ Carseview centre, 4 Tom McDonald Avenue, Dundee, UK. DD2 1NH. \\ ${ }^{2}$ Professor of Psychiatry and Psychotherapy, University of Bonn, Germany \\ *email: nikudhillonanhs.net
}

DOI: 10.2478/gp-2018-0007

Received: 28 August 2018; Accepted: 28 August 2018

\begin{abstract}
Objectives: Clozapine is an effective antipsychotic medication licenced for the management of treatment resistant schizophrenia. Due to its non-selective pharmacology, it has a broad range of side effects. Nocturnal enuresis secondary to the use of clozapine has been documented in the literature but may be overlooked, the link between drug and symptom being clinically unnoticed. Patients may not mention urinary symptoms due to supervening psychosis, co-existing symptomatology, embarrassment or shame. By raising awareness of the phenomenon, early recognition and patient support may improve compliance with clozapine medication, and consecutively, overall mental health. Consequently, this systematic review investigates the prevalence of nocturnal enuresis secondary to clozapine use.

Methods: A literature search on clozapine and nocturnal enuresis was used to identify the relevant papers. Papers providing the prevalence data on Clozapine associated nocturnal enuresis were selected for data extraction.

Results: 47 papers were initially identified. Eight papers focused on the prevalence of clozapine associated nocturnal enuresis (CANE). Point prevalence (nocturnal enuresis at the time of assessment), 1-month prevalence and episode prevalence (nocturnal enuresis since beginning of clozapine) were given. Papers included patients with schizophrenia, schizoaffective disorder, bipolar affective disorder and psychotic depression, taking clozapine medication. The prevalence of CANE ranged from 10-42\%. Point prevalence was 21-27\%, 1-month prevalence was 10-39\% and episode prevalence was 15-42\%. Clozapine was more likely to cause nocturnal enuresis compared to other psychotropic medication.

Conclusion: The prevalence of CANE may be greater than previously thought. However, in order to determine an accurate prevalence of clozapine associated nocturnal enuresis, larger studies with strict inclusion criteria, common definition of diagnosis and prevalence are required. By establishing an accurate prevalence, physician awareness can be improved, and patients can be counselled on the risk of developing the side effect, thus improving early identification and reducing discontinuation rates.
\end{abstract}

\section{Keywords}

Clozapine, schizophrenia, nocturnal enuresis, urinary incontinence, antipsychotic, systematic review, side effect.

\section{INTRODUCTION}

Schizophrenia is a lifelong psychiatric disorder that impairs normal functioning. Patients can present with positive, negative or disorganised symptoms. Despite modern pharmacological advances, $20-30 \%$ of patients do not respond to first or second line treatments (Miyamoto et al. 2014). Such treatment resistant patients usually experience profound disability including selfneglect, social isolation and suicide. The efficacy and side effect profile of available antipsychotics for the treatment of schizophrenia vary substantially (Leucht et al. 2013).

The atypical antipsychotic, clozapine, was first developed in 1961 and introduced in Europe in 1970 (De Fazio et al. 2015). It marked a major advancement in psychiatry due to its effective management of negative symptoms in schizophrenia, in addition to reducing suicidality without the increased burden of extra-pyramidal side effects. Clozapine has been shown to be superior in efficacy with $50-60 \%$ of patients with previous treatment resistant schizophrenia responding to the medication (Lally et al. 2015). As a result of clozapine's broad and concerning side effect profile, it is prescribed only when other psychotropic medications have been tried and exhausted. As the relationship between mental and physical health is an intricate one, it is important for physicians to be familiar with the side effects of the medication.

Clozapine was subsequently withdrawn in 1975 after seventeen patients experienced myelosuppression and eight of them died as a result of neutropenic sepsis (De Fazio et al. 2015). Albeit 
rare, an overwhelming infection is a well-recognised side effect. It is also important that physicians, particularly psychiatrists and general practitioners who frequently encounter clozapine prescriptions are also aware of the other non-life threatening side effects, which have an impact on patients' quality of life. Clozapine's non-selective pharmacological profile can cause a multitude of side effects as a result of anti-serotoninergic, anti-dopaminergic, anti-adrenergic, anti-muscarinic and antihistaminergic actions (De Fazio et al. 2015).

Clozapine associated nocturnal enuresis (CANE) is a symptom that carries social stigma and may not be easy for a patient to discuss. Only if the physician is aware of the phenomenon can the symptom be sought by direct questioning. Compared to daytime incontinence, the presence of nocturnal enuresis is less likely to be volunteered as a result of the associated embarrassment, and so, patients may suffer in silence or even discontinue the medication. By raising awareness, physicians might better identify and manage the condition and gather more accurate data on prevalence. This would allow early recognition, patient education and management. Compliance would be improved, thus improving mental health and reducing the need for inpatient admission.

To assess the relevance of clozapine associated nocturnal enuresis, the aim of this systematic review was to determine the prevalence of nocturnal enuresis secondary to clozapine use.

\section{METHODS}

\section{Literature search}

To investigate the prevalence of nocturnal enuresis in patients taking clozapine, a systematic review was conducted using the electronic data source, PubMed. The literature search was performed in March 2018. The search terms used were 'clozapine', 'nocturnal enuresis' and 'urinary incontinence. Those articles that could not be accessed through OpenAthens were requested through a departmental librarian.

\section{Article selection}

Papers that addressed clozapine and nocturnal enuresis were of interest. The inclusion criteria were:

- Relevant articles should report prevalence and could include cross sectional, prospective or cohort studies.

- Patients must be taking clozapine regardless of dose.

- All major psychiatric diagnoses were included.

- Articles that included patients taking other concurrent medications were included.
Exclusion criteria were:

- As this review specifically analysed clozapine's effect on nocturnal enuresis, articles that only examined daytime urinary incontinence and other lower urinary tract symptoms were excluded that examined daytime urinary incontinence and other lower urinary tract symptoms.

- Review articles and case reports were excluded as they did not explore the prevalence of clozapine associated nocturnal enuresis

- Non-English papers were excluded as a result of our limited language skills.

\section{Data extraction}

Data was extracted from the selected papers: author, year of publication, study type, country of study, sample size, age and gender, diagnostic instruments used, clozapine dose, the use of concurrent medications, frequency of nocturnal enuresis, study conclusions and study limitations.

\section{RESULTS}

Figure 1 describes the literature search and its outcome.

Eight articles were included in the review.

- Two studies assessed the CANE point prevalence (nocturnal enuresis at the time of assessment) using a cross sectional approach (Centorrino et al. 1994, Long et al. 2015).

- Two studies provided a 1 month prevalence using a retrospective approach (Jeong et al. 2008, Yusufi et al. 2007)

○ A Jeong et al. 2008 assessment was part of a 2-year prospective follow-up study.

- Four studies assessed the CANE episode prevalence (nocturnal enuresis since beginning of clozapine) using a retrospective approach (Bhirud, Shah. 2004, HarrisonWoolrych et al. 2011, Lin et al. 1999 and Warner et al. 1994).

- Harrison-Woolrych et al. 2011 retrospective assessment was part of an observational cohort study

\section{General and demographical data}

The prevalence of clozapine associated nocturnal enuresis ranged from $10-42 \%$ (see table 1). Point prevalence was $21-$ $27 \%, 1$-month prevalence was $10-39 \%$ and episode prevalence was $15-42 \%$.

The studies included a range of psychiatric diagnoses from schizophrenia, schizoaffective disorder, bipolar affective 


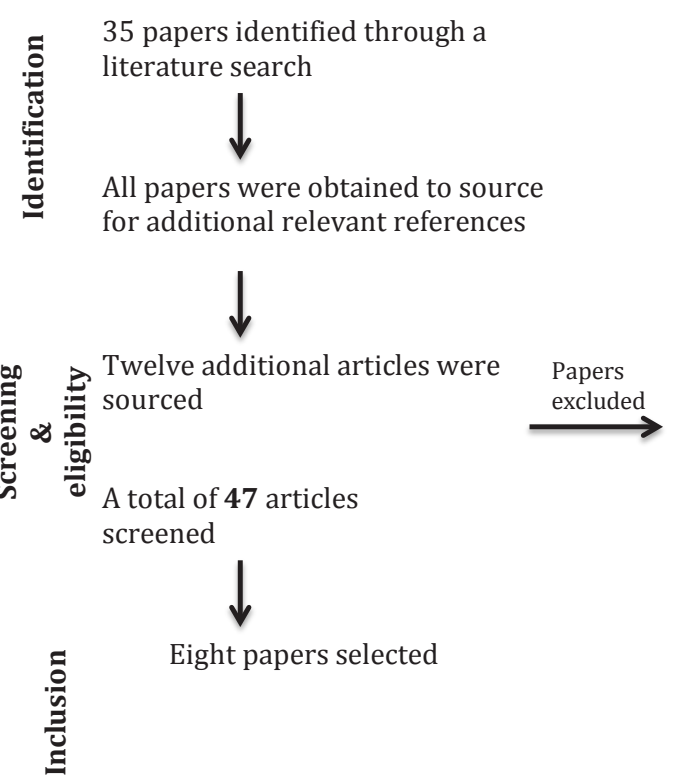

- Two papers were in other languages one in French and one in German.

- Seven were review articles

- 16 were case reports

- Five were not relevant to the prevalence of nocturnal enuresis in clozapine use

- Four focused on daytime urinary incontinence as a side effect to clozapine

- Three used clozapine to manage urinary incontinence

- One was a comment on a retrospective study

- One publication was a guideline

Figure 1. Literature search and screening

disorder, psychotic depression and obsessive compulsive disorder. Only Lin et al. (1999) and Warner et al. (1994) exclusively explored the side effect in patients with schizophrenia alone. Their point prevalence was 41 and $42 \%$ respectively.

Study populations were of both inpatient and outpatient groups. There did not appear to be any significant difference between the prevalence of nocturnal enuresis in those patients taking clozapine in the inpatient compared to the outpatient setting (see table 1).

The study sample sizes included in this review ranged from 12 to 103 . The study populations were predominantly male, ranging from $60-75 \%$ male, aside from the cross sectional by Long et al. (2015), which involved female inpatients from a secure psychiatric facility for women.

Generally, the age ranges of the studies were 16-64 years. Long et al. (2015) included patients up to the age of 70 years old. Mean age ranged from 37 to 41.5 years.

Jeong et al. (2008) observed a higher frequency of nocturnal enuresis in women compared to men. This study was comprised of $60 \%$ males. In contrast, Lin et al. (1999) did not find any significant difference between men and women experiencing nocturnal enuresis.

\section{Course and presentation of nocturnal enuresis}

Bhirud et al. (2004) observed that participants experienced the symptom either in the first three weeks of initiation or when the dose was increased. Warner et al. (1994) observed that all participants experienced NE within 12 weeks of clozapine initiation regardless of dose.

Two studies described the natural course of CANE as either self-limiting or persistent. Bhirud et al. (2004) observed that $20 \%$ of patients self-resolved within an unspecified time and the remainder resolved after an intervention of either dose reduction or with the use of imipramine. Whereas, Warner et al. (1994) observed full resolution of NE in all twelve patients within three months.

Of the patients that admitted to nocturnal enuresis few volunteered the information, highlighting the importance of direct questioning. Yusufi et al. (2007) observed that one in 40 patients who spontaneously reported the symptom of $\mathrm{NE}$ on initial general questioning compared to 39 out of 40 positive responses on direct questioning regarding NE. Warner et al. (1994) observed that four out of the five patients that experienced NE did not disclose the symptom during the initial general patient interview, possibly due to embarrassment.

\section{Clozapine dosage and concurrent medications}

Six studies elaborated on the mean clozapine dose (see table 1). Yusufi et al. (2007) observed a weak but significant association between clozapine dose and severity of side effects. However, this was not specific to nocturnal enuresis. No other studies elaborated on the relationship between clozapine dose and presence of NE. 


\begin{tabular}{|c|c|c|c|c|c|}
\hline 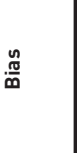 & & 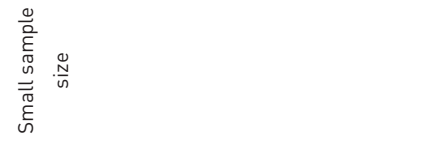 & 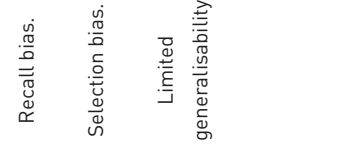 & & 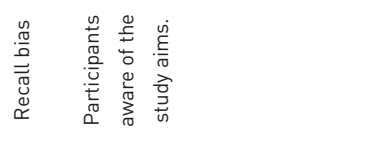 \\
\hline & & 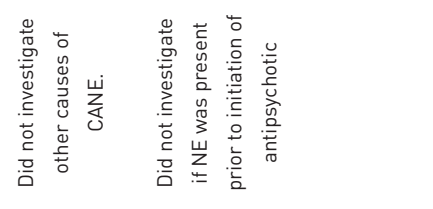 & 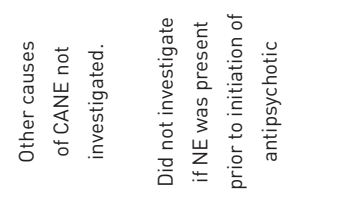 & & 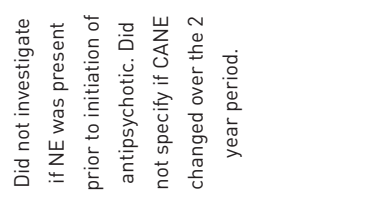 \\
\hline & & 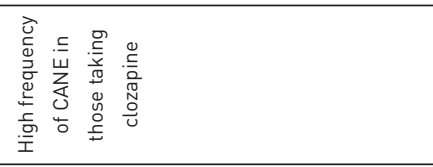 & 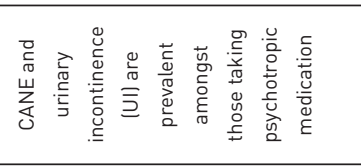 & & 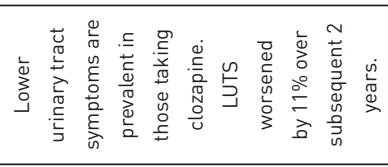 \\
\hline 离 & & 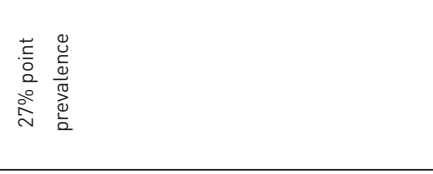 & 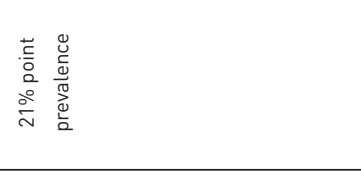 & & 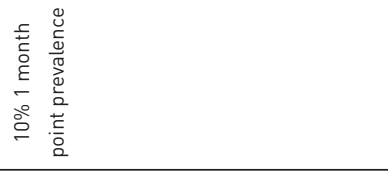 \\
\hline 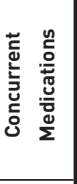 & & 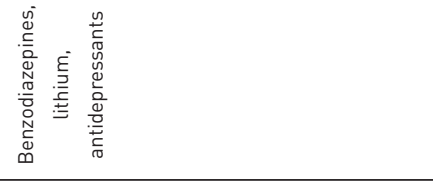 & 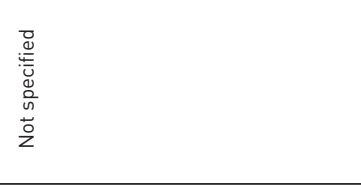 & & 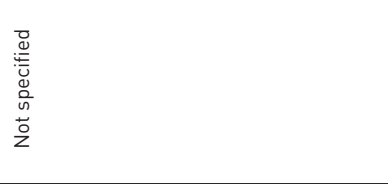 \\
\hline 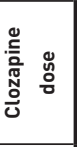 & & 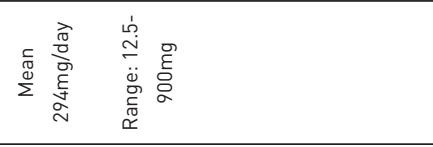 & 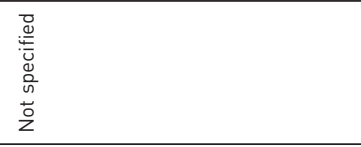 & & 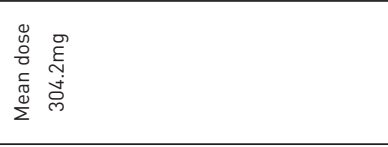 \\
\hline 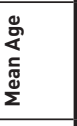 & & 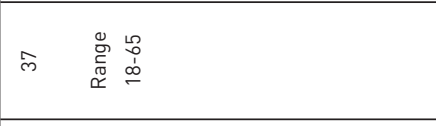 & 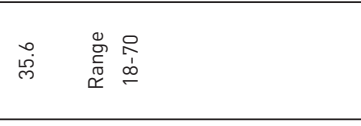 & & 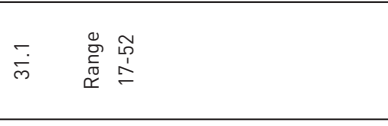 \\
\hline 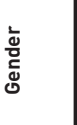 & & 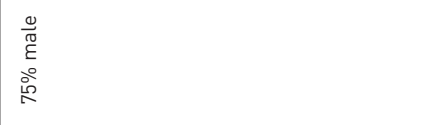 & 离 & & 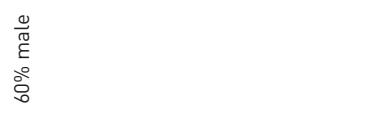 \\
\hline 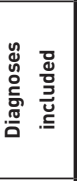 & & 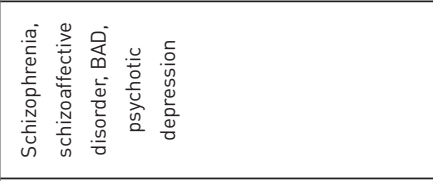 & 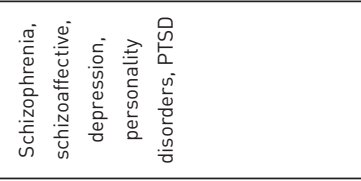 & & 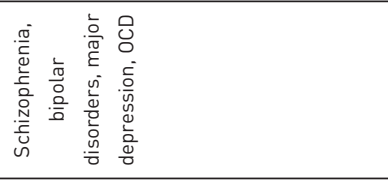 \\
\hline 产 & & 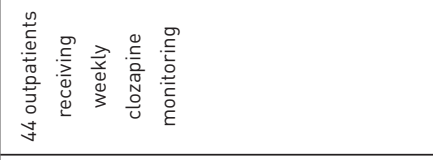 & 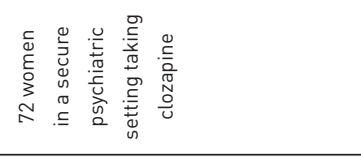 & & 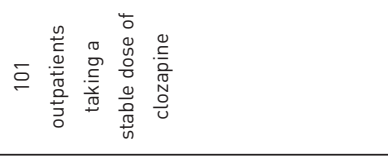 \\
\hline 咅 & & 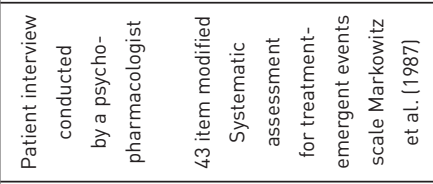 & 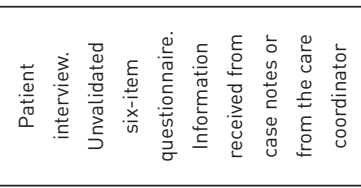 & & 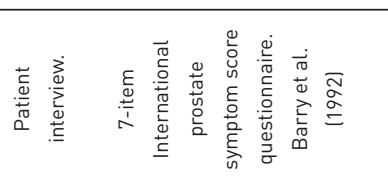 \\
\hline 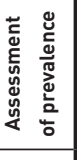 & & 怘 & 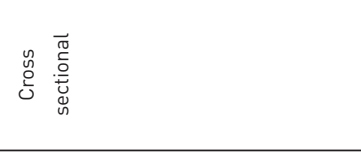 & & 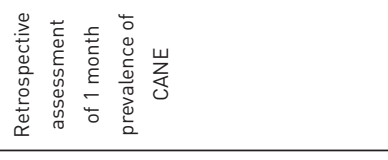 \\
\hline 咅 & & 昏 & 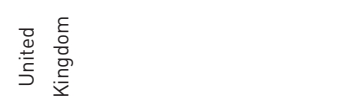 & & 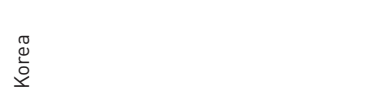 \\
\hline 吾 & 䓂 & 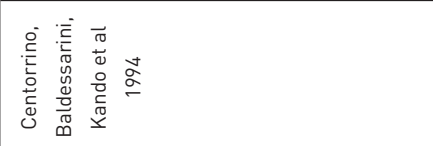 & 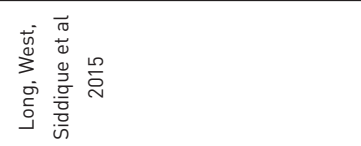 & 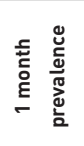 & 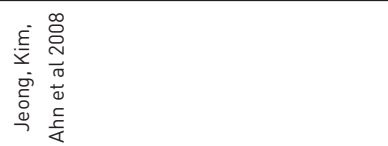 \\
\hline
\end{tabular}




\begin{tabular}{|c|c|c|c|c|}
\hline$\frac{\mathrm{n}}{\mathrm{m}}$ & 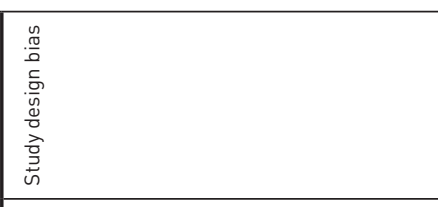 & & 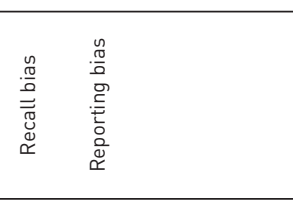 & 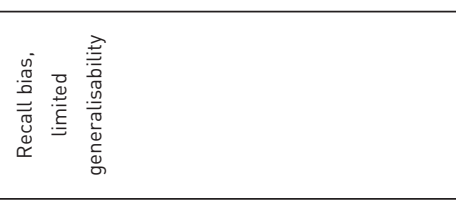 \\
\hline 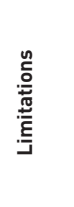 & 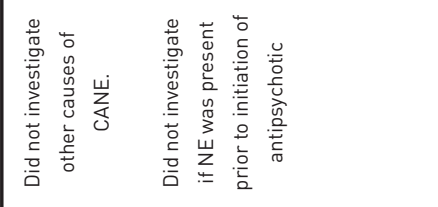 & & 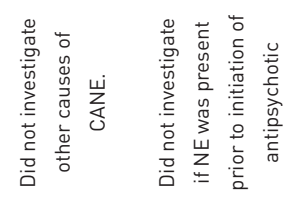 & 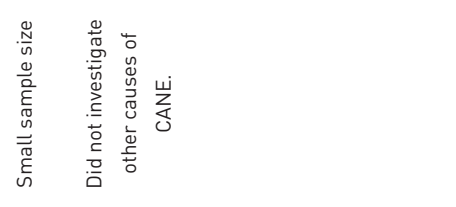 \\
\hline 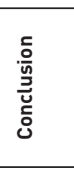 & 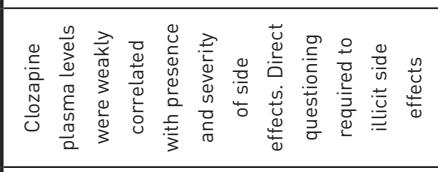 & & 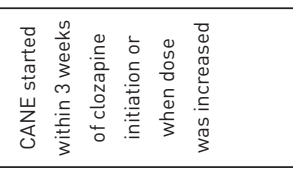 & 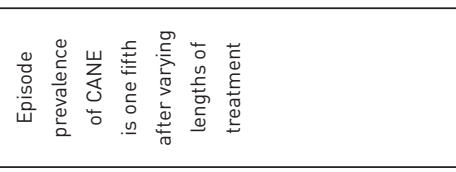 \\
\hline 离 & 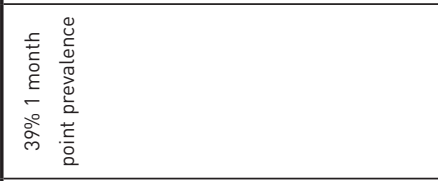 & & 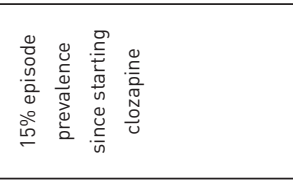 & 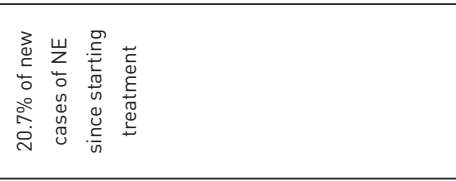 \\
\hline 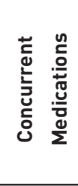 & 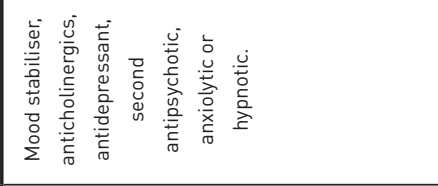 & & 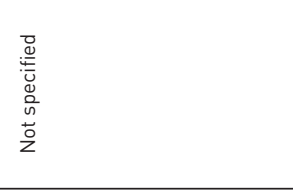 & 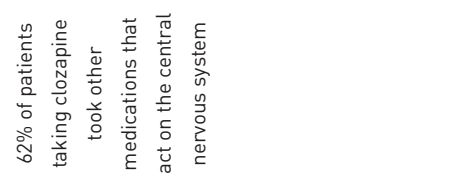 \\
\hline 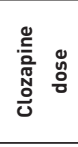 & 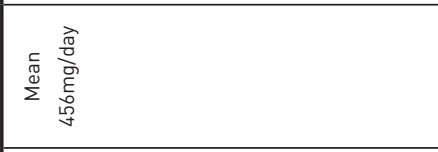 & & 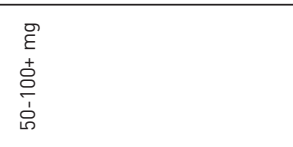 & 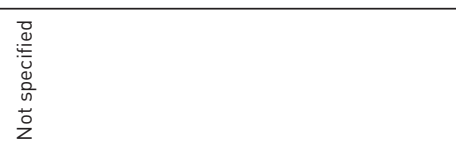 \\
\hline 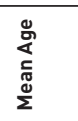 & 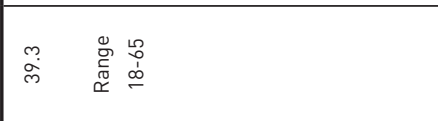 & & 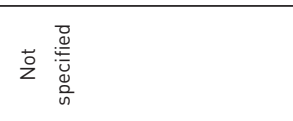 & 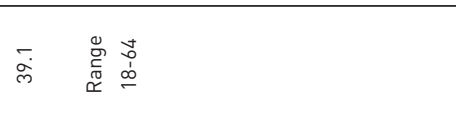 \\
\hline 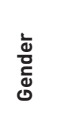 & 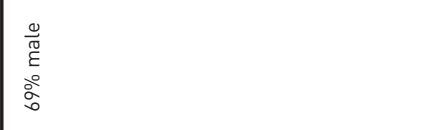 & & 总 & 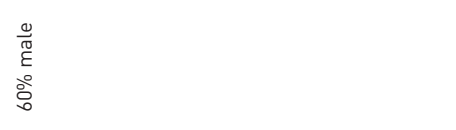 \\
\hline 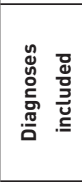 & 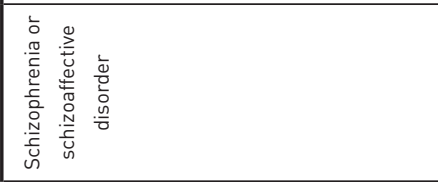 & & 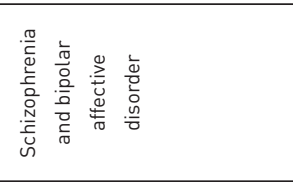 & 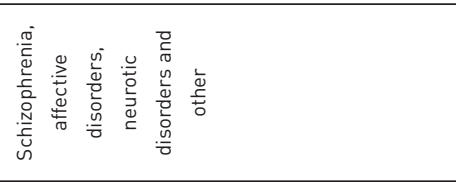 \\
\hline 递 & 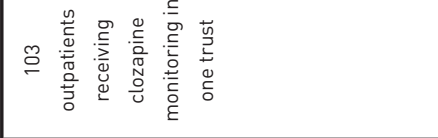 & & 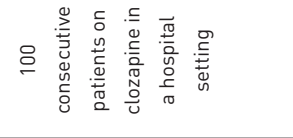 & 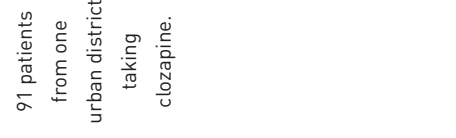 \\
\hline 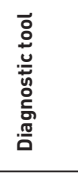 & 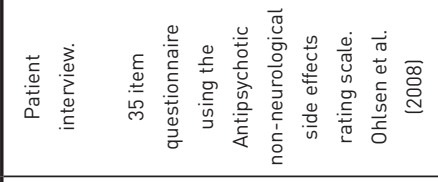 & & 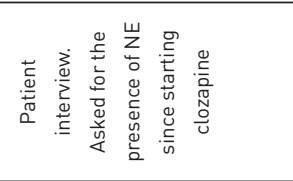 & 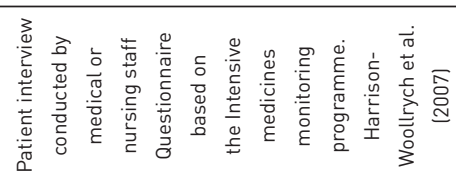 \\
\hline 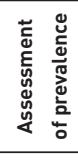 & 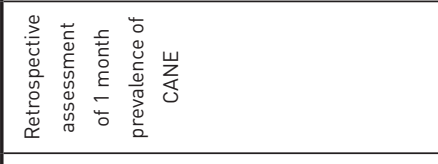 & & 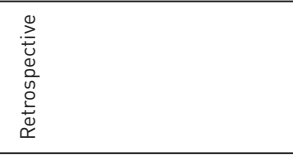 & \\
\hline 䇏 & 总高 & & $\underline{\underline{\underline{\underline{w}}}}$ & 蛋总 \\
\hline 誉离 & 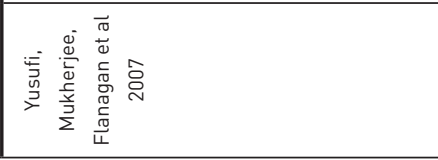 & 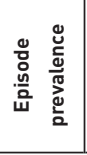 & 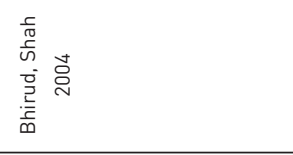 & 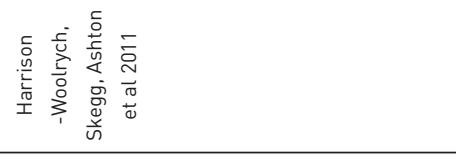 \\
\hline
\end{tabular}




\begin{tabular}{|c|c|c|}
\hline 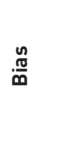 & 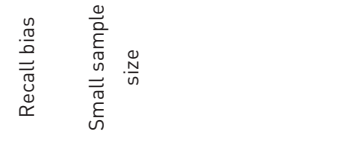 & 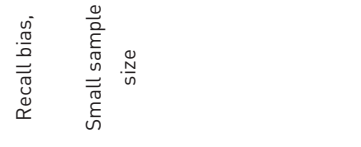 \\
\hline & 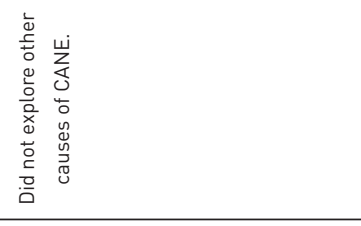 & 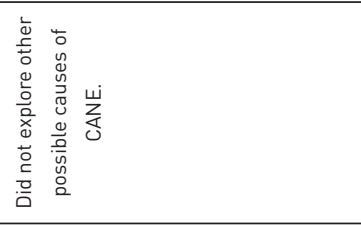 \\
\hline 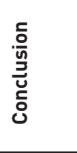 & 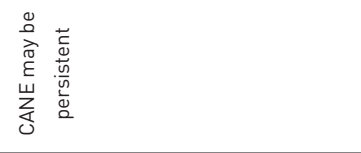 & 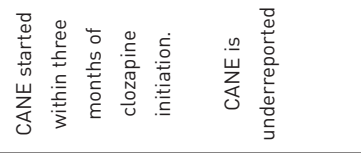 \\
\hline 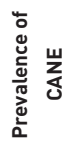 & 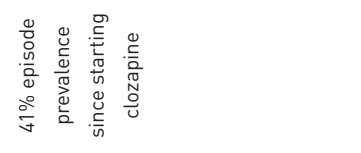 & 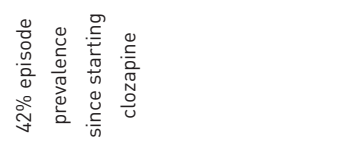 \\
\hline 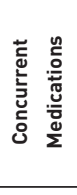 & 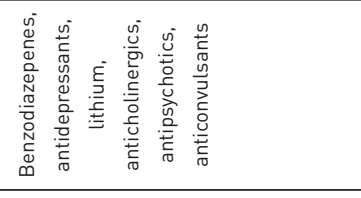 & 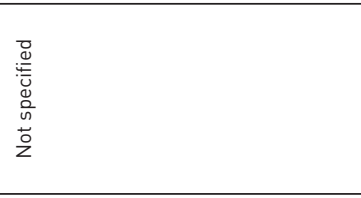 \\
\hline 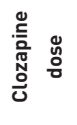 & 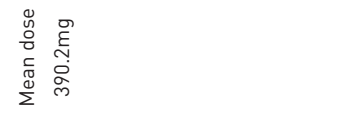 & 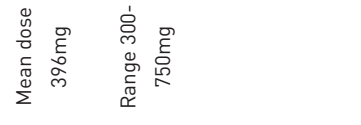 \\
\hline 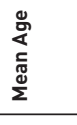 & ô & $\stackrel{n}{\stackrel{\leftrightarrow}{\forall}}$ \\
\hline 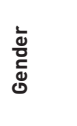 & 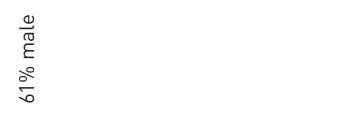 & 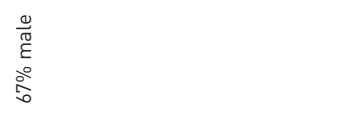 \\
\hline 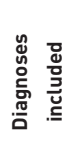 & 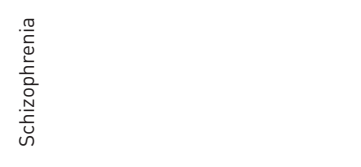 & 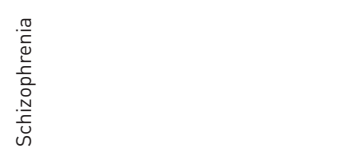 \\
\hline 总 & 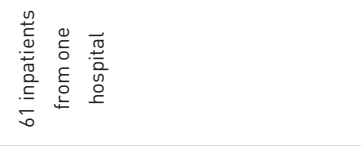 & 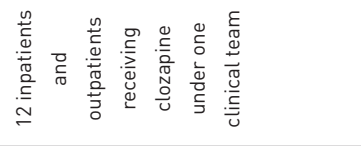 \\
\hline 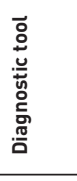 & 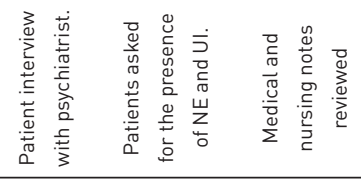 & 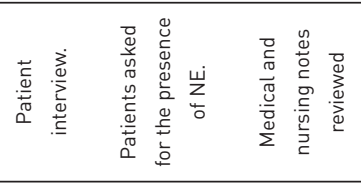 \\
\hline 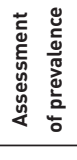 & 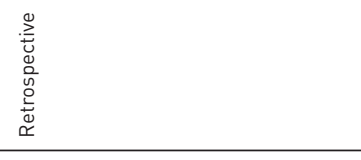 & 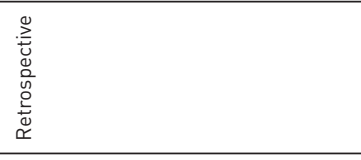 \\
\hline $\begin{array}{l}\underline{\underline{z}} \\
\text { 咅 }\end{array}$ & 莺 & 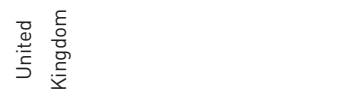 \\
\hline 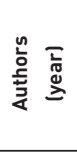 & 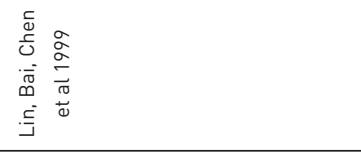 & 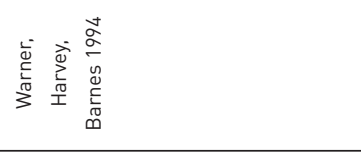 \\
\hline
\end{tabular}


Four studies (Centorrino et al. 1994, Yusufi et al. 2007, Harrison-Woolrych et al. 2011 and Lin et al. 1999) specified the use of concurrent medications in their sample, which included centrally acting medications, for example, antipsychotics, antidepressants, antimuscarinics and benzodiazepines. The remaining four studies did not specify if concurrent medications were excluded. No study explored the prevalence of nocturnal enuresis in patients on clozapine alone.

On comparison with the other antipsychotics, clozapine was more likely to cause nocturnal enuresis. HarrisonWoolyrch et al. (2011) described a prevalence of clozapine associated nocturnal enuresis to be $20.7 \%$ compared to $9.6 \%$, $6.7 \%$ and $6.2 \%$ with olanzapine, quetiapine and risperidone respectively. Long et al. (2015) also observed that clozapine was more likely to cause nocturnal enuresis when compared to the other antipsychotics.

\section{Risk of bias and limitations across studies}

Bias in the study design

All studies included patients on varying lengths of clozapine treatment. Three studies (Harrison-Woolrych et al. 2011, Long et al. 2015 and Bhirud et al. 2004) did not exclude patients based on a minimal duration of treatment. The remaining studies included patients on a stable maintenance phase of clozapine treatment. By only including the patients on an established dose, some cases of NE may have been missed, thus underestimating the prevalence. For example, those patients who had discontinued clozapine during the initiation phase due to NE itself. And patients who had experienced self-resolving NE may not have reported the symptom due to inaccurate recollection. This may be the case in studies that aimed to achieve point prevalence.

Six studies did not exclude patients with a previous history of urinary incontinence prior to clozapine initiation. Jeong et al. (2008) and Lin et al. (1999) aimed to reduce the rate of false positive findings by incorporating past medical history into the exclusion criteria.

Only Long et al. (2015) endeavoured to further investigate patient's urinary incontinence but not specifically nocturnal enuresis to reduce the rate of false positives. Long et al. described that half of all the patients who experienced urinary incontinence received a urine dipstick and $85 \%$ were referred to a GP to exclude other organic causes.

\section{Bias}

Potential of Recall bias in studies included

All studies that utilised a questionnaire based patient interview approach were exposed to recall bias, as it heavily relied on accurate patient recollection and timing of symptom events. Particularly the studies that reported an episode prevalence since clozapine initiation. This applied to both patients and healthcare staff when asked to clarify the presence or absence of NE.

Generally, the studies did not explicitly document the time period over which the presence of NE was assessed during the patient interview, that is, whether the symptom was experienced currently, within the last week, since clozapine initiation or at any point over their lifetime. Jeong et al. (2008) and Yusufi et al. (2007) clearly documented that the questions were asked in relation to the past month.

\section{Ascertainment bias}

Ascertainment bias was present in the four studies that had used unvalidated questionnaires as their diagnostic tool. All the studies included a face-to-face patient interview, although the person who conducted the interview was not always specified. Centorrino et al. (1994), Lin et al. (1999) and Harrison-Woolrych et al. (2011) utilised the experience of trained psychopharmacologists, psychiatrists and mental health nurses to perform the interviews (see table 1).

\section{Lack of standardisation and generalisability}

There were differences in how the prevalence was evaluated. Four studies determined the prevalence by relying on accurate patient recall since starting clozapine. The remaining four studies used a point or 1-month point prevalence.

\section{DISCUSSION}

This is the first systematic review to investigate the prevalence of clozapine associated nocturnal enuresis. The prevalence ranged from $10-42 \%$ and compared to other antipsychotics clozapine was more likely to cause NE. Point prevalence was $21-27 \%$, 1-month prevalence was $10-39 \%$ and episode prevalence was $15-42 \%$.

Sagy et al. (2014) has cited a prevalence of CANE to be as low as $0.23 \%$ by Sandoz, a finding which remains unpublished and so cannot be validated. The large range of the prevalence is likely to be attributable to the differing study design, inclusion criteria and 
diagnostic tools used to determine the presence of NE. The highest rates were found in the studies that included smaller sample sizes and inpatient populations, suggesting that the severity of mental illness may have an effect on the presence of NE.

\section{Inpatient and outpatient populations}

No previous studies have compared the rate of CANE between the inpatient and outpatient populations. Although it is difficult to compare the studies as a result of differing study designs, there does not appear to be a significant difference between the patient populations (see table 1).

\section{Other side effects of clozapine}

Compared to the other side effects, nocturnal enuresis seems to be relatively prevalent. Among clozapine's other side effects, sedation and hypersalivation are the most commonly experienced affecting $70 \%$ and $57 \%$ of patients, respectively (Yusufi et al. 2007). Sexual dysfunction and nocturnal enuresis, although not often disclosed, occur with a higher frequency (55\% and $39 \%$ ) ahead of other more well recognised side effects, such as constipation and weight gain (34\% and 31\%) (Yusufi et al. 2007). In future studies, it would be of benefit to examine the rate of daytime urinary incontinence associated with clozapine use.

\section{Nocturnal enuresis in the general population}

The prevalence of NE in the population taking clozapine medication is considerably higher than the general population. A telephone survey of 8,500 adults aged 16-40 years by Yeung et al. (2008) found the prevalence of nocturnal enuresis in the general population to be over $2 \%$. This review estimates that the prevalence of clozapine associated nocturnal enuresis to be $10-42 \%$. As the patient population who require clozapine have risk factors for urinary incontinence, including psychosis itself, it is difficult to ascertain an accurate prevalence of true CANE. Hsu et al. (2007) conducted a study involving 30,000 participants and discovered that the patients with schizophrenia had a 1.78 fold risk increase of urinary incontinence compared to the patients without schizophrenia after accounting for co-morbidities and medication.

\section{Criteria needed to establish CANE prevalence in future studies}

From the assessed publications, it was difficult to ascertain if clozapine was the primary cause for nocturnal enuresis, as no single study clearly meets the above mentioned criteria to arrive at an accurate prevalence. Although only three studies' aims (Harrison-Woolrych et al. 2011, Bhirud et al. 2004 and Warner et al. 1994) were to determine the prevalence of clozapine associated nocturnal enuresis, a more rigorous patient selection and prospective design would help ascertain a true prevalence.

In order to give a true prevalence of CANE, we suggest future studies to incorporate the following criteria:

- Nocturnal enuresis must have developed after clozapine initiation.

- Other causes for NE should be explored such as the use of diuretics, presence of a urinary tract infection, urge and stress incontinence, benign prostatic hypertrophy, epilepsy, cognitive impairment, neurological disability, diabetes mellitus, diabetes insipidus and psychosis itself. Only Long et al. (2015) attempted to explore the alternative causes of new incontinence. Clozapine associated nocturnal enuresis should only be considered once the mentioned have been excluded.

- The diagnostic tool was used to determine the presence of nocturnal enuresis. Those studies that relied solely on patient recall had less power than those that used a validated questionnaire during the interview setting.

- The temporal relationship between clozapine initiation and the timing of NE development is important as a symptom that develops soon after an intervention is more likely to be due to the intervention.

- To produce a true prevalence, all patients should ideally be prescribed clozapine alone. However, as co-morbidity and polypharmacy are common in patients taking clozapine medication, it can be difficult to ascertain the cause and effect. To reduce confounding, the four studies highlighted relevant co-existing medications. The articles reviewed on NE caused by concurrent use of both psychiatric and non-psychiatric medications were few, thus reducing the impact of a true prevalence.

To elicit the prevalence of CANE in future studies, a prospective approach should be adopted. Patients should be identified prior to clozapine initiation and subsequently interviewed at regular intervals post-initiation. As clozapine initiation often requires inpatient management, it would be possible to enquire about specific symptoms frequently during medicine rounds before clozapine is dispensed, similar to how routine physical observations are recorded. Although, it may add to workload and additional checklists, it is important to recognise such side effects early in order to improve compliance and overall mental health. 


\section{Mechanism of CANE}

The cause of nocturnal enuresis secondary to clozapine use is considered to be multifactorial. A collection of three case studies by Kho et al. (2011) describes how nocturnal enuresis developed through differing mechanisms. Clozapine's sedative effect was observed in a female patient with schizophrenia who was found to sleep too deeply to empty her bladder. The seizure threshold is also lowered by the antipsychotic and nocturnal seizures may cause incontinence. All antipsychotics, in particular clozapine, increase insulin resistance and polyuria secondary to diabetes may cause incontinence. To further confound the clinical presentation, incontinence may be a symptom of psychosis itself (Warner et al. 1994.).

The precise mechanism of how clozapine affects the bladder is under speculation. Clozapine's anti-cholinergic effect on the detrusor muscle and anti-adrenergic effect on the urethral sphincter are demonstrated by the following case reports.

Cohen et al. (1994) describes the case of a 38 year old man with paranoid schizophrenia who developed nocturnal enuresis secondary to urinary retention. He was subsequently catheterised, and four litres of urine was drained. It was believed that clozapine's strong anti-cholinergic effect was attributable to the urinary retention.

Fuller et al. (1996) managed clozapine induced daytime urinary incontinence with an alpha agonist, ephedrine, in order to counteract clozapine's anti-adrenergic effect on the internal bladder sphincter. Of the 16 cases that were treated with ephedrine, twelve had complete resolution of symptoms and three had partial improvement. The participants were aware of the study aims and the presence of incontinence was determined either by urine stained linen or patient complaint.

\section{Clozapine to treat urinary incontinence}

It is worth noting that three papers were excluded in the screening process as clozapine was observed to treat urinary incontinence. Balhara et al. (2011) describe a case study where the use of clozapine was used to successfully manage a schizophrenia patient's urinary incontinence. However, on clozapine titration, the urinary incontinence reappeared when the dose exceeded $300 \mathrm{mg}$ per day. Kumar et al. (2007) and Mathew et al. (1996) describe similar cases. It is likely that incontinence was attributable to psychosis, which subsequently improved with clozapine. And the reason for the reoccurrence at higher doses of clozapine may be due to clozapine induced urinary incontinence.

\section{CONCLUSION}

Nocturnal enuresis is an under-reported and under recognised side effect of clozapine. It impacts negatively on quality of life and contributes to reduced compliance in a patient population that already experiences management difficulties. The lifethreatening side effects of clozapine are well-recognised; however, greater awareness of lesser known side effects are still needed. Future studies with a prospective design, greater sample size and carefully selected control groups are still needed to establish a true prevalence of clozapine associated nocturnal enuresis.

Declaration of interest: None of the authors have any interest to declare in relation to this paper.

Ethical approval and informed consent not required for systematic literature review.

\section{REFERENCES}

Balhara Y. Clozapine and bladder control. $\mathrm{Br} J$ Psychiatry. 2011;199(6):518-9.

Barry MJ, Fowler FJ Jr, O'Leary MP, Bruskewitz RC, Holtgrewe HL, Mebust WK, Cockett AT. The American Urological Association symptom index for benign prostatic hyperplasia. The Measurement Committee of the American Urological Association. J Urol. 1992;148(5):1549-57; discussion 1564.

Bhirud M, Shah N. Clozapine induced urinary incontinence during sleep. Indian J Psychiatry. 2004;46(3):280.
Centorrino F, Baldessarini RJ, Kando JC, Frankenburg FR, Volpicelli SA, Flood JG. Clozapine and metabolites: concentrations in serum and clinical findings during treatment of chronically psychotic patients. J Clin Psychopharmacol. 1994;14(2):119-25.

Cohen MA, Alfonso CA, Mosquera M. Development of urinary retention during treatment with clozapine and meclizin. Am J Psychiatry. 1994;151(4):619-20.

De Fazio P, Gaetano R, Caroleo M, Cerminara G, Maida F, Bruno A, Muscatello MR, Moreno MJ, Russo E, Segura-García C. Rare and 
very rare adverse effects of clozapine. Neuropsychiatr Dis Treat. 2015;11:1995-2003.

Frankenburg FR, Kando JC, Centorrino F, Gilbert JM. Bladder dysfunction associated with clozapine therapy. J Clin Psychiatry. 1996;57(1):39-40.

Fuller MA, Borovicka MC, Jaskiw GE, Simon MR, Kwon K, Konicki PE. Clozapine-induced urinary incontinence: incidence and treatment with ephedrine. J Clin Psychiatry. 1996;57(11):514-8.

Harrison-Woolrych M, Coulter DM. PEM in New Zealand. In Pharmacovigilance (eds R Mann, E Andrews): 317-32. John Wiley \& Sons, 2007.

Harrison-Woolrych M, Skegg K, Ashton J, Herbison P, Skegg DC Nocturnal enuresis in patients taking clozapine, risperidone, olanzapine and quetiapine: comparative cohort study. $\mathrm{Br} \mathrm{J}$ Psychiatry. 2011;199(2):140-4.

Hsu WY, Muo CH, Ma SP, Kao CH. Association between schizophrenia and urinary incontinence: A population-based study. Psychiatry Res. 2017;248:35-39.

Jeong SH, Kim JH, Ahn YM, Lee KY, Kim SW, Jung DC, Kim YS. A 2-year prospective follow-up study of lower urinary tract symptoms in patients treated with clozapine. J Clin Psychopharmacol. $2008 ; 28(6): 618-24$

Kho KH, Nielson O. Clozapine-induced nocturnal enuresis: diagnostic and treatment issues. Psych Bulletin. 2001;25:232-233.

Kumar A, Chadda RK, Kumar N, Balan S, Prakash Srinivasan S. Clozapine for the treatment of neurogenic bladder. Neurourol Urodyn. 2007;26(4):537-539.

Lally J, MacCabe JH. Antipsychotic medication in schizophrenia: a review. Br Med Bull. 2015;114(1):169-79.

Leucht S, Cipriani A, Spineli L et al. Comparative efficacy and tolerability of 15 antipsychotic drugs in schizophrenia: a multipletreatments meta-analysis. Lancet. 2013;382(9896):951-62.

Lin CC, Bai YM, Chen JY, Lin CY, Lan TH. A retrospective study of clozapine and urinary incontinence in Chinese in-patients. Acta Psychiatr Scand. 1999;100(2):158-61.

Long CG, West R, Siddique R, Rigg S, Banyard E, Stillman SK, Butler $S$, Dolley 0 . Screening for incontinence in a secure psychiatric service for women. Int J Ment Health Nurs. 2015;24(6):451-9.

Markowitz JS, Rabkin JG, Stewart J, Tricamo E, McGrath PJ, Harrison W, Ocepek-Welikson K, Quitkin FM. Validity assessment of SAFTEE: a preliminary report. Psychopharmacol Bull. 1987;23(1):102-5.
Mathew VM, Dursun SM, Reveley MA. Successful treatment of urinary incontinence with clozapine in a schizophrenic patient. J Psychopharmacol. 1996;10(2):166-9.

Miyamoto S, Jarskog LF, Fleischhacker WW. New therapeutic approaches for treatment-resistant schizophrenia: a look to the future. J Psychiatr Res. 2014;58:1-6.

Ohlsen RI, Williamson R, Yusufi B, Mullan J, Irving D, Mukherjee S, Page E, Aitchison KJ, Barnes TR. Interrater reliability of the Antipsychotic Non-Neurological Side-Effects Rating Scale measured in patients treated with clozapine. J Psychopharmacol. 2008;22(3):323-9.

Sagy R, Weizman A, Katz N. Pharmacological and behavioral management of some often-overlooked clozapine-induced side effects. Int Clin Psychopharmacol. 2014 Nov;29(6):313-7.

Warner JP, Harvey CA, Barnes TR. Clozapine and urinary incontinence. Int Clin Psychopharmacol. 1994;9(3):207-9.

Yeung CK, Sihoe JDY, Sit FKY, Bower W, Sreedhar B, Lau JTF. Epidemiologicalstudy of primarynocturnalenuresis(bed-wetting) in young adults in Hong Kong Hong. Kong Med J. 2008;14/Suppl 3): $\$ 28-30$

Yusufi B, Mukherjee S, Flanagan R, Paton C, Dunn G, Page E, Barnes TR. Prevalence and nature of side effects during clozapine maintenance treatment and the relationship with clozapine dose and plasma concentration. Int Clin Psychopharmacol. 2007;22(4):238-43. 